\title{
The subcellular localization of PBX1 and EXD proteins depends on nuclear import and export signals and is modulated by association with PREP1 and HTH
}

\author{
Jens Berthelsen, ${ }^{2,3}$ Charlotte Kilstrup-Nielsen, ${ }^{1,3}$ Francesco Blasi, ${ }^{2}$ Fulvio Mavilio, ${ }^{1}$ and \\ Vincenzo Zappavigna ${ }^{1,4}$ \\ ${ }^{1}$ TIGET-Laboratory of Gene Expression, and ${ }^{2}$ Molecular Genetics Unit, Dipartimento di Ricerca Biologica e Tecnologica, \\ H.S. Raffaele, 20132 Milan, Italy
}

\begin{abstract}
Nuclear localization of the Extradenticle (EXD) and PBX1 proteins is regionally restricted during Drosophila and mammalian development. We studied the subcellular localization of EXD, PBX, and their partners Homothorax (HTH) and PREP1, in different cell contexts. HTH and PREP1 are cytoplasmic and require association with EXD/PBX for nuclear localization. EXD and PBX1 are nuclear in murine fibroblasts but not in Drosophila Schneider cells, in which they are actively exported to the cytoplasm. Coexpression of EXD/PBX with HTH/PREP1 causes nuclear localization of their heterodimers in both cell contexts. We propose that heterodimerization with HTH/PREP induces nuclear translocation of EXD and PBX1 in specific cell contexts by blocking their nuclear export.
\end{abstract}

[Key Words: Homeodomain; gene expression; developmental regulation; protein-protein interaction; nuclear import; nuclear export]

Received December 4, 1998; revised version accepted February 26, 1999.

The PBC subfamily, of homeodomain proteins includes the products of the vertebrate $P b \times 1, P b \times 2$, and $P b \times 3$, the Drosophila extradenticle (exd), and the Caenorhabditis elegans ceh-20 genes (Burglin 1997). The exd gene plays a crucial role during fly embryogenesis, cooperating with the genes of the Homeotic Complex (HOM-C) in the specification of segmental identities (for review, see Mann and Chan 1996). Similarly, the vertebrate PBX proteins display cooperative binding with HOX proteins (Mann and Chan 1996), allowing the selective activation of promoters containing HOX/PBX binding sites (Di Rocco et al. 1997; Maconochie et al. 1997). We recently reported that PBX proteins form stable heterodimers with the novel homeodomain protein PREP1 (Berthelsen et al. 1998a). Prep1 belongs to a novel subclass of the TALE superfamily of homeobox genes (Burglin 1997) and is related to the mammalian Meis and Drosophila homothorax (hth) gene products (Moskow et al. 1995; Rieckhof et al. 1997; Berthelsen et al. 1998b; Pai et al. 1998). Their amino acid sequence conservation extends beyond the homeodomain and comprises two domains, HR1 and HR2, located within the amino terminus of the three proteins (Burglin 1997; Berthelsen et al. 1998b). The HR1

\footnotetext{
${ }^{3}$ These authors contributed equally to this work.

${ }^{4}$ Corresponding author.

E-MAIL ZAPPAV@TIGEM.IT; FAX 39-02-26434668.
}

and HR2 regions were shown to be essential for interaction between PREP1 (Berthelsen et al. 1998a), or MEIS1 (Knoepfler et al. 1997) and the PBC proteins. Similarly, PBC-family proteins share two conserved regions in their amino-terminal portion, termed PBC-A and PBC-B (Burglin and Ruvkun 1992). In PBC proteins, the surface necessary to contact PREP1 or MEIS1 is located within the conserved PBC-A domain (Chang et al. 1997; Knoepfler et al. 1997; Berthelsen et al. 1998a). Unlike PBC and Hox proteins, however, PREP1 and PBX interact efficiently in the absence of DNA (Berthelsen et al. 1998b). Likewise, MEIS1 was shown to form complexes with PBX1 in the absence of DNA, and to bind DNA cooperatively with PBX1 (Chang et al. 1997; Knoepfler et al. 1997).

The Drosophila Meis-homolog hth was recently found to act, in cooperation with exd, as a master antennaldetermining gene in Drosophila development (Casares and Mann 1998). Additionally, $h$ th was shown to interact with exd in patterning the embryonic peripheral nervous system (Pai et al. 1998), and to suppress eye development of the fly (Kurant et al. 1998). HTH is thought to exert its action by inducing EXD nuclear localization (Rieckhof et al. 1997). During Drosophila embryogenesis, in the absence of coexpressed HTH, EXD is found to be mainly localized to the cytoplasm, whereas only nuclear EXD reveals to be functional (Rieckhof et al. 1997; Kurant et al. 1998; Pai et al. 1998). The molecular 
mechanism leading to nuclear localization of the two proteins, however, remains to be elucidated. It is still unknown whether the cytoplasmic localization of EXD is due to the lack of a nuclear localization signal (NLS), which might be provided by HTH, or whether HTH antagonizes a mechanism that segregates EXD in the cytoplasm. It is also still unknown whether EXD is required for nuclear localization of HTH (see Kurant et al. 1998).

Also in vertebrates, PBX protein function appears to be regulated at the level of subcellular localization. PBX displays a pattern of subcellular distribution in the developing mouse limb buds that is reminiscent of that observed in the Drosophila leg imaginal disc, because PBX is cytoplasmic in cells located in more distal regions of the limb buds (Gonzales-Crespo et al. 1998). However, the role of the mammalian PREP1 and MEIS1 proteins in the regulation of subcellular localization of PBX proteins has never been directly addressed.

We have investigated the mechanisms underlying the regulation of subcellular localization of PBX1 and EXD proteins, and the role played by PREP1 and HTH in this process, in different cell contexts. We show that in mouse fibroblasts, PREP1 is localized mainly to the cytoplasm, whereas PBX1 is localized to the nucleus. In Drosophila Schneider cells, PBX1 or EXD, as well as PREP1 or HTH, are all found in the cytoplasm. Coexpression of $P b x 1$ with Prep 1 or $h t h$, and of exd with $h t h$ or Prep1, leads to nuclear localization of the respective proteins in both cell contexts. PBX1 contains a NLS, located within its homeodomain, whose activity in Schneider cells is negatively regulated by the PREP/ HTH heterodimerization surface. Treatment of transfected Schneider cells with an inhibitor of receptor-mediated nuclear export, induces nuclear accumulation of PBX1 and EXD, indicating that their cytoplasmic localization is based on export from the nucleus. We propose that complex formation with PREP/MEIS/HTH proteins triggers nuclear translocation of $\mathrm{PBC}$ proteins by preventing the activity of a nuclear export signal located within the interaction surface between PBC and PREP/ MEIS/HTH proteins.

\section{Results and Discussion}

PREP1 requires interaction with $P B X 1$ for nuclear localization

When NIH-3T3 cells were transiently transfected with a construct expressing the human $P b x 1$ cDNA under the control of the SV40 promoter, the subcellular localization of the produced PBX1 protein was exclusively nuclear, as detected by immunofluorescence staining (Fig. 1A). Conversely, transfection with a construct expressing Prep1 showed that only a minor fraction of the produced PREP1 protein is localized to the nucleus, whereas most of it is found in the cytoplasm of transfected cells (Fig. 1B). Because we showed previously that PREP1 is found mainly associated with PBX proteins (Berthelsen et al. 1998b), we tested whether coexpression with PBX1 would induce PREP1 nuclear import. As shown in Figure $1(\mathrm{C}, \mathrm{D})$, cotransfection of $P b x 1$ together with Prep1 resulted in nuclear localization of PREP1, as revealed by immunofluorescence staining. Confocal analysis showed that PREP1 and PBX1 wholly colocalize within the nuclei of coexpressing cells (data not shown). Identical results were obtained transfecting other mammalian cell lines such as COS7 or HeLa cells (data not shown).

To test whether direct interaction between PBX1 and PREP1 is necessary for nuclear localization of PREP1, we transfected a mutant derivative of PREP1, PREP1 $\triangle H R 1+2$, which lacks the two conserved amino-terminal domains, HR1 and HR2, required for interaction with PBX1 (Berthelsen et al. 1998b). The PREP1 $\Delta$ HR $1+2$ mutant protein was found in the cytoplasm of expressing cells as its wild-type counterpart, but was not translocated into nuclei if coexpressed with PBX1 (summarized in Table 1). Similarly, the PBX1 $\Delta 1-140$ mutant (Di Rocco et al. 1997), representing a deletion of the amino-terminal PBC-A domain of PBX1, which is necessary for heterodimerization with PREP1 (Berthelsen et al. 1998a,b), was unable to trigger nuclear localization of PREP1 (Fig. $1 F)$. The PBX $\Delta 1-140$ mutant was nuclear as the wildtype PBX1 (Fig. 1E,G). These results were confirmed by immunoblot analysis of protein extracts from transfected COS7 cells (data not shown). Overall, these data indicate that nuclear localization of PREP1 requires the formation of a PBX1-PREP1 complex, because deletion of the interaction surfaces on either protein is sufficient to abolish nuclear translocation.

Recent reports showed that the EXD protein is localized to the cytoplasm in specific subsets of cells during fly development, and that coexpression of HTH was associated with nuclear localization of EXD (Rieckhof et al. 1997; Pai et al. 1998). Thus, we wondered whether the cytoplasmic localization of EXD in Drosophila cells in the absence of HTH was due to an intrinsic property of EXD, different from PBX1, or was rather controlled by some cell context-dependent mechanism. When expressed in NIH-3T3 mouse fibroblasts, EXD, in the absence of HTH, was found only in the nuclei of expressing cells, whereas HTH, like its related vertebrate protein PREP1, was found in the cytoplasm (see Table 1). Coexpression of EXD and $\mathrm{HTH}$, induced their colocalization to the nuclei of NIH-3T3 cells (Table 1). Thus, EXD, like PBX1, is translocated to the nuclei of mouse fibroblasts without coexpressed HTH, whereas HTH, like PREP1, requires the coexpression of EXD for nuclear translocation.

\section{The NLS of the PBX1-PREP1 complex is located within the PBX1 homeodomain}

We wanted to define the regions within the PBX1-PREP1 complex required for its nuclear localization. As NLSs of homeodomain proteins were shown in most cases to reside within the homeodomain (for review, see Derossi et al. 1998), we generated a deletion mutant of PBX1, PBX1NT, lacking the entire homeodomain and carboxyl terminus of the protein. As shown in Figure $1 \mathrm{H}$, the 

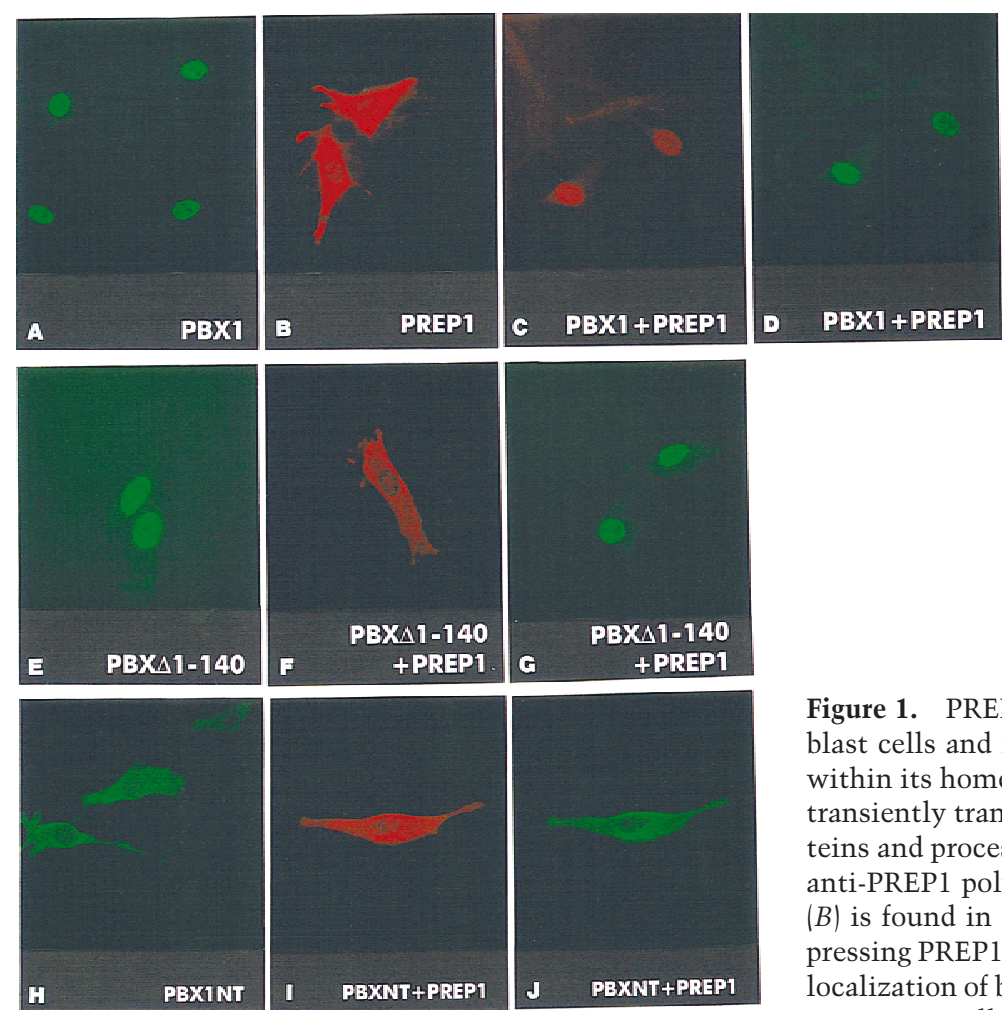

Figure 1. PREP1 is found in the cytoplasm of transfected mouse fibroblast cells and requires interaction with PBX1, which possesses a NLS within its homeodomain, for nuclear translocation. NIH-3T3 cells were transiently transfected with expression constructs for the indicated proteins and processed for indirect immunofluorescence with anti-PBX1 or anti-PREP1 polyclonal antibodies. PBX1 is nuclear $(A)$, whereas PREP1 $(B)$ is found in the cytoplasm of expressing NIH-3T3 cells. Cells coexpressing PREP1 and PBX1 (in red and green, respectively) display nuclear localization of both proteins $(C, D)$. The $\mathrm{PBX} \Delta 1-140$ mutant is nuclear in expressing cells $(E, G$, green), but is unable to trigger nuclear translocation of PREP1 $(F$, red). A PBX1 mutant lacking the homeodomain, PBXNT, is cytoplasmic in expressing NIH-3T3 cells ( $H$, green). When coexpressed, PREP1 (red) and PBXNT (green) are both found in the cytoplasm of NIH-3T3 cells ( $I$ and $J$, respectively). Fusions of PREP1 with the SV40 NLS (NLS-PREP1), or with the PBX1 homeodomain (PREP/ $\mathrm{PBXHD})$ are nuclear $(K, L$, red). The reciprocal chimeric protein PBX/ PREPHD is found in the cytoplasm ( $M$, green). Double stainings $(C, D, I, J)$ were performed with anti-HA rat monoclonal, to detect HA-tagged PBX1 and PBXNT, and anti-PREP1 polyclonal antibodies.

PBX1NT protein was found exclusively in the cytoplasm of expressing cells. Moreover, the PBX1NT mutant, although being able to associate with PREP1 in vitro (data not shown), was unable to induce nuclear localization of PREP1 (Fig. 1I,J). Conversely, deletion mutants lacking either the carboxyl terminus, PBX1 $2296-430$, or the amino terminus, PBX $\Delta 1-230$ (Di Rocco et al. 1997) of PBX1, were found in the nuclei (data not shown), indicating that the NLS of PBX1 is located within its homeodomain.

Recent studies on the subcellular distribution of EXD in Drosophila (Rieckhof et al. 1997; Pai et al. 1998) showed that HTH is required for nuclear localization of EXD. From these results it could be assumed that in the absence of HTH, or its vertebrate homologs MEIS1 and PREP1, EXD/PBX proteins would be unable to translocate into the cell nucleus. In transfected murine fibroblasts, as well as in other differentiated mammalian cells (COS7, HeLa), instead, we always observed nuclear localization of PBX1 or EXD in the absence of PREP1 or $\mathrm{HTH}$, even if expressed at high levels. This suggests that in several cell contexts PBC proteins may not necessarily require the coexpression of $\mathrm{PREP} / \mathrm{HTH} / \mathrm{MEIS}$ proteins for their nuclear transport, because they possess a functional NLS.

Next, we tested whether the PREP1 protein could be translocated into the nucleus in the absence of PBX1, following fusion with a NLS. We generated two derivatives of PREP1, the first carrying the NLS of the SV40 large $\mathrm{T}$ antigen, NLS-PREP1, and the second, PREP1/ PBXHD, replacing the homeodomain/carboxyl terminus of PREP1 with the corresponding region of PBX1. As shown in Figure $1 \mathrm{~K}, \mathrm{~L}$, both NLS-PREP1 and the PREP1/ PBXHD chimera were nuclear in expressing cells. Conversely, the reciprocal chimeric mutant, carrying a substitution of the homeodomain/carboxyl terminus region of PBX1 with the corresponding region of PREP1, PBX1/ PREPHD, was localized solely to the cytoplasm (Fig. $1 \mathrm{M})$. These results show that providing the PREP1 protein with a NLS is sufficient to cause its nuclear translocation in the absence of PBX1, and suggest that the cytoplasmic localization of PREP1 is likely due to the lack of a functional NLS. A short stretch of basic amino acids (RRKRR), resembling the basic stretch of the SV40 NLS (Kalderon et al. 1984), is present at the amino terminus of the PBX1 homeodomain (Kamps et al. 1990) but 
Table 1. Subcellular localization of PBX, EXD, PREP1, $\mathrm{HTH}$, and their mutant derivatives

\begin{tabular}{|c|c|c|c|}
\hline & & Loc & ation \\
\hline Protein & & $\begin{array}{l}\text { mouse } \\
\text { fibroblast }\end{array}$ & $\begin{array}{c}\text { Drosophila } \\
\text { Schneider } \\
\text { cells }\end{array}$ \\
\hline $\mathrm{PBX} 1$ & & $\mathrm{~N}$ & $\mathrm{C}$ \\
\hline EXD & & $\mathrm{N}$ & $\mathrm{C}$ \\
\hline PREP1 & & $\mathrm{C}$ & $\mathrm{C}$ \\
\hline НTH & & $\mathrm{C}$ & $\mathrm{C}$ \\
\hline PBX1 + PREP1 & & $\mathrm{N}+\mathrm{N}$ & $\mathrm{N}+\mathrm{N}$ \\
\hline $\mathrm{EXD}+\mathrm{HTH}$ & & $\mathrm{N}+\mathrm{N}$ & $\mathrm{N}+\mathrm{N}$ \\
\hline EXD + PREP1 & & $\mathrm{N}+\mathrm{N}$ & $\mathrm{N}+\mathrm{N}$ \\
\hline PBX1 + HTH & & $\mathrm{N}+\mathrm{N}$ & $\mathrm{N}+\mathrm{N}$ \\
\hline PREP1 $\Delta \mathrm{HR} 1+2$ & & $\mathrm{C}$ & N.D. \\
\hline PBX $1+$ PREP $1 \Delta$ & $\mathrm{HR} 1+2$ & $\mathrm{~N}+\mathrm{C}$ & N.D. \\
\hline NLS-PREP1 & & $\mathrm{N}$ & N.D. \\
\hline PBX/PREPHD & & $\mathrm{C}$ & N.D. \\
\hline PREP/PBXHD & & $\mathrm{N}$ & N.D. \\
\hline PBX/PREPHD + & PREP/PBXHD & $\mathrm{N}+\mathrm{N}$ & N.D. \\
\hline PBXNT & & $\mathrm{C}$ & N.D. \\
\hline PBXNT + PREP & & $\mathrm{C}+\mathrm{C}$ & N.D. \\
\hline NLS-PREP1 + P & BXNT & $\mathrm{N}+\mathrm{N}$ & N.D. \\
\hline NLS-PREP1 + P & XX/PREPHD & $\mathrm{N}+\mathrm{N}$ & N.D. \\
\hline PBX $\Delta 1-72$ & & N.D. & $\mathrm{C}$ \\
\hline PBX $\Delta 1-90$ & & N.D. & $\mathrm{N}$ \\
\hline $\mathrm{PBX} 1 \Delta 1-140$ & & $\mathrm{~N}$ & $\mathrm{~N}$ \\
\hline $\mathrm{PBX} \Delta 1-72+\mathrm{PR}$ & & N.D. & $\mathrm{N}+\mathrm{N}$ \\
\hline $\mathrm{PBX} \Delta 1-72+\mathrm{HT}$ & & N.D. & $\mathrm{N}+\mathrm{N}$ \\
\hline $\mathrm{PBX} 1 \Delta 1-140+\mathrm{P}$ & REP1 & $\mathrm{N}+\mathrm{C}$ & N.D. \\
\hline PBX 1 & $\begin{array}{l}\text { PBC-A PBC-B } \\
\text { WZZAEZA- }\end{array}$ & & \\
\hline PBX $1 \Delta 1-72$ & EAEA & & \\
\hline PBX $1 \Delta 1-90$ & GEA & & \\
\hline PBX $1 \Delta 1-140$ & $\pi$ & & \\
\hline PBX NT & एய2पWD & & \\
\hline PREP1 & $\begin{array}{l}\text { HR1 HR2 } \\
\text { WEX }\end{array}$ & & \\
\hline PREP $1 \Delta \mathrm{HR} 1+2$ & & & \\
\hline NLS-PREP1 & & & \\
\hline PREP/PBX HD & 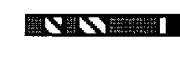 & & \\
\hline PBX/PREP HD & WWAWLOAD: & & \\
\hline
\end{tabular}

Summary of the subcellular localizations of the PBX1, EXD, PREP1, and HTH proteins and their mutant derivatives (schematically represented below), in NIH-3T3 mouse fibroblasts and in Schneider cells. (N) Nuclear localization; (C) cytoplasmic localization; (N.D.) not determined.

is not conserved in the PREP1 homeodomain (Berthelsen et al. 1998b).

To prove that nuclear localization of the PREP1-PBX1 complex requires interaction between the two proteins and the presence of a NLS, we coexpressed PBX1NT with NLS-PREP1, or PREP1/PBXHD with PBX1/PREPHD, or NLS-PREP1 with PBX1/PREPHD. All combinations resulted in nuclear localization of the coexpressed proteins (see Table 1). These data further confirm that PBX1 and
PREP1 translocate to the nucleus as a protein complex. PREP1 lacks a NLS, but is located into the nucleus in mouse fibroblasts (or in COS and HeLa cells) following heterodimerization with PBX1, which conversely possesses a NLS. These results also rule out the possibility that nuclear translocation of PREP1 is triggered via an indirect action of $P b x 1$, for example, by inducing the expression of an endogenous gene product.

A subregion of the conserved PBC-A domain mediates the cytoplasmic localization of PBX1 in Drosophila Schneider cells

To investigate the role of the cellular context in the cytoplasmic localization of EXD, we transfected Drosophila Schneider cells (SL-2) with constructs expressing exd, hth, and their vertebrate counterparts $P b x 1$ and Prep1. EXD was shown to be cytoplasmic and to be translocated to the nucleus if coexpressed with MEIS1 (Rieckhof et al. 1997; and this work; see Table 1). Schneider cells thus represent a good model to investigate the regulation of subcellular localization of EXD/ PBX and HTH/PREP1 proteins.

We found HTH to be localized essentially to the cytoplasm of transfected Schneider cells. Following coexpression, EXD and HTH are both translocated to the cell nuclei (Table 1). PBX1 or PREP1 were also found in the cytoplasm of transfected cells (Fig. 2A,B). Coexpression of PBX1 and PREP1 similarly induced nuclear localization of both proteins (Fig. 2C,D). Coexpression of EXD with PREP1, and of PBX1 with HTH resulted in nuclear transport of the corresponding complexes, as in the case of NIH-3T3 cells (see Table 1), indicating that EXD and PBX1 are interchangeable for heterodimerization with HTH or PREP1, and for triggering their nuclear transport. Thus PBX1, like its Drosophila counterpart EXD, is cytoplasmic in Schneider cells and translocates into the nucleus only following coexpression with either PREP1 or HTH. In Drosophila Schneider cells, as in mouse fibroblasts, the Drosophila EXD and HTH, and their mammalian counterparts PBX1 and PREP1, display the same subcellular localization, and can replace each other to form nuclear heterodimers (see Table 1).

To understand the mechanism of cytoplasmic localization of EXD and PBX1 in Schneider cells, we tested the subcellular distribution of $\operatorname{PBX} 1 \Delta 1-140$, lacking the conserved amino-terminal PBC-A domain. Surprisingly, the PBX $\Delta 1-140$ mutant protein was found exclusively in the nuclei of expressing cells (Fig. 2E), indicating that the $\mathrm{PBC}-\mathrm{A}$ domain, which is required for interaction with the PREP1/MEIS1/HTH proteins, is also mediating cytoplasmic localization of the PBX1 protein in Schneider cells. The nuclear localization of PBX $\Delta 1-140$ further indicates that the PBX1 NLS is potentially functional also in Schneider cells, but its activity is suppressed by sequences within the PBC-A domain.

To further restrict the region required for cytoplasmic localization of PBX1 in Schneider cells, we generated two additional deletion mutants of PBX1: one carrying a deletion of the first 72 amino acids, $\operatorname{PBX} \Delta 1-72$, and the 


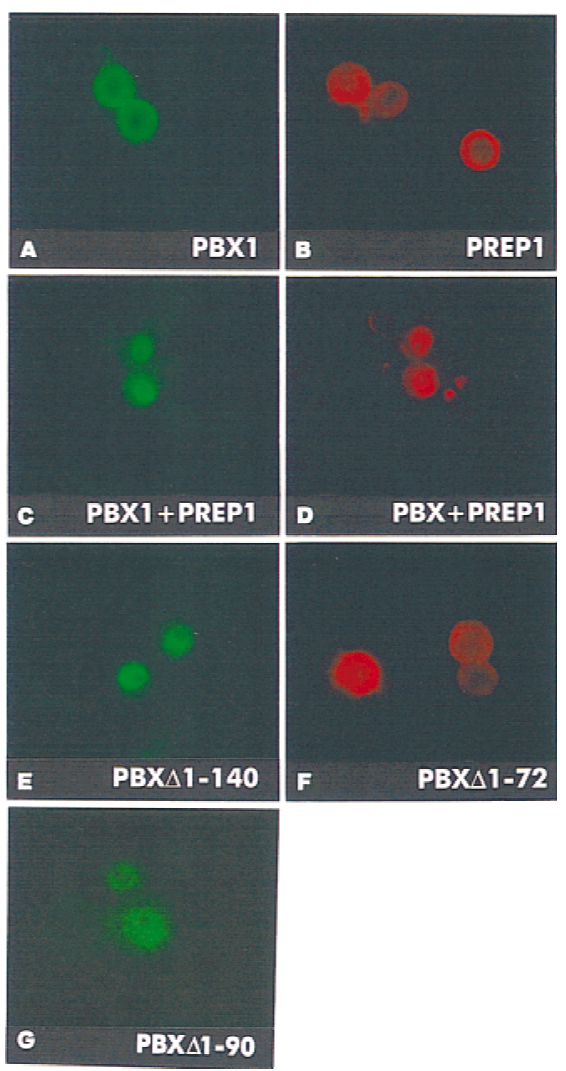

Figure 2. The EXD/PBX1 and HTH/PREP1 proteins are mutually required for nuclear localization in transfected Drosophila Schneider cells. A subregion of the PBC-A domain is necessary for cytoplasmic localization of PBX1 in Schneider cells. Drosophila Schneider cells were transiently transfected with expression constructs for the indicated proteins, and processed for indirect immunofluorescence with anti-PREP1 and anti-PBX1 polyclonal, or anti-FLAG and anti-HA monoclonal antibodies. PBX1 ( $A$, green) and PREP1 $(B$, red) are found in the cytoplasm of expressing Schneider cells. Coexpression of PREP1 with PBX1 (C, green) causes nuclear localization of PREP1 (D, red). A PBX1 mutant, representing a deletion of its amino-terminal PBC-A domain, PBX $\Delta 1-140$ ( $E$, green), is nuclear in Schneider cells in the absence of PREP1 or HTH. In contrast, the PBX $\Delta 1-72$ mutant $(F$, red) is found in the cytoplasm. PBX $\Delta 1-90$ is nuclear in expressing cells $(G$, green). Double stainings $(C, D)$ were performed with anti-HA (rat) monoclonal, and anti-PREP1 polyclonal antibodies.

other, PBX $\Delta 1-90$, a deletion of the first 90 amino acids. As shown in Figure 2, the PBX $\Delta 1-72$ protein was detected in the cytoplasm (F), whereas PBX $\Delta 1-90$ was nuclear in expressing cells $(G)$, indicating that the region spanning amino acids 73-90 within the PBC-A domain is required for cytoplasmic localization of PBX1 in Schneider cells. Following coexpression with PREP1 or $\mathrm{HTH}$, the PBX $\Delta 1-72$ mutant protein was found in the nucleus (see Table 1), indicating that deletion of the first 72 amino acids of PBX1 does not impair complex formation with PREP1 or HTH. Conversely, PBX $\Delta 1-90$, representing a deletion identical to that of the E2A-PBX1 fusion oncogene (Kamps et al. 1990), does not interact with
MEIS1 or PREP1 (Knoepfler et al. 1997; Berthelsen et al. 1998a; data not shown).

These results indicate that a stretch of 18 amino acids within the conserved PBC-A domain is required both for interaction with PREP1/MEIS1/HTH proteins, and for cytoplasmic localization of PBX1 in Schneider cells. Our results further indicate that in Drosophila Schneider cells there is a mutual requirement between $\mathrm{PBC}$ and PREP1/HTH proteins for nuclear translocation: EXD/ PBX1 require HTH/PREP1 to overcome a cell context -dependent block of NLS activity, whereas HTH/PREP1, as in mouse fibroblasts, likely require a functional NLS to translocate into the nucleus. Both conditions are fulfilled following the formation of a heterodimer between members of the two protein families.

\section{The cytoplasmic localization of EXD and PBX in Schneider cells is mediated by nuclear export}

To explain the cytoplasmic localization of PBX and EXD in Schneider cells, despite the presence of a NLS, we considered the possibility that PBX and EXD might be actively exported from the nucleus. In eukaryotic cells, nuclear export is mediated by receptors recognizing specific nuclear export signals (NESs) within their cargoes (for review, see Ohno et al. 1998). A receptor mediating nuclear export of proteins containing leucine-rich NES, exportin1 (CRM1), was recently identified in yeast and vertebrate cells, and its activity was shown to be specifically inhibited by the cytotoxin leptomycin B (LMB) in yeast, Xenopus, and mammalian cells (for review, see Mattaj and Englmeier 1998). We treated Schneider cells, transfected with the expression constructs for PBX1 or EXD, with increasing amounts of LMB. As shown in Figure $3 \mathrm{~A}$, whereas $5 \mathrm{nM}$ LMB had no effect on the subcellular localization of PBX1, $50 \mathrm{~nm}$ or $250 \mathrm{~nm}$ LMB caused efficient nuclear accumulation of PBX1 in Schneider cells. Similarly, EXD (Fig. 3B) and PBX $\Delta 1-72$ (Fig. 3C), were found in the nuclei of cells treated with $50 \mathrm{~nm}$ or $250 \mathrm{~nm}$ LMB. These results show that the cytoplasmic localization of PBX1 and EXD in Schneider cells is based on LMB-sensitive nuclear export. In contrast, PREP1 did not accumulate in the nuclei of expressing Schneider cells at all tested concentrations of $\operatorname{LMB}(50,250$, and $500 \mathrm{~nm}$, data not shown). Because relatively large globular proteins are unable to cross the nuclear pores by diffusion (for review, see Ohno et al. 1998), this result further confirms that the cytoplasmic localization of PREP1 $(64 \mathrm{kD})$ is due to the lack of a NLS, and passive exclusion from the nucleus.

Because PBX $\Delta 1-140$ and PBX $\Delta 1-90$ were spontaneously nuclear, whereas PBX $\Delta 1-72$ was exported to the cytoplasm, we can conclude that the region comprising amino acids 73-90 of PBX1 contains sequences that are required for its nuclear export. The PBC-A subdomain of PBX1 spanning amino acids 73-90 contains three leucine residues, which are conserved among $\mathrm{PBC}$ family members, and may thus represent a target for nuclear export receptor recognition. Active nuclear export of PBC proteins appears to be cell context-dependent, because ex- 


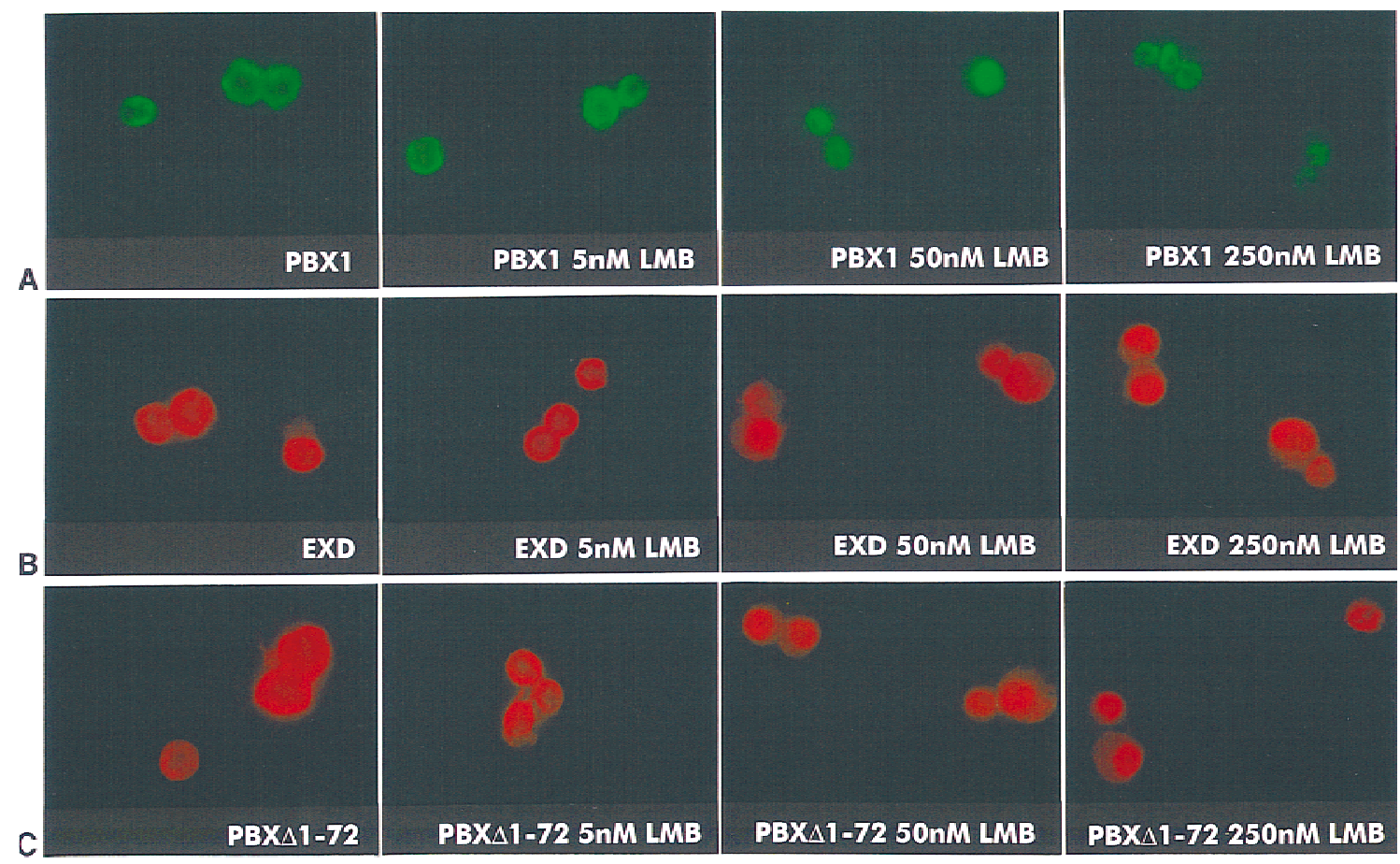

Figure 3. LMB blocks nuclear export of PBX1, EXD, and PBX $1-72$ proteins in Drosophila Schneider cells. Cells were transiently transfected with expression constructs for PBX1, EXD, and PBX $\Delta 1-72$ and processed for indirect immunofluorescence with anti-PBX1 and anti-Flag antibodies. Schneider cells, transfected with the PBX1, the EXD or the PBX $\Delta 1-72$ expressors were treated with 5, 50, and $250 \mathrm{nM}$ LMB as indicated. Fifty and $250 \mathrm{nM} \mathrm{LMB}$ induce relocalization of PBX1 (A, green), of EXD (B, red), or of the PBX $\Delta 1-72 \mathrm{mutant}$ $(C$, red) into the nuclei of expressing cells. Treatment with LMB at the indicated concentrations was performed $18 \mathrm{hr}$ after transfection.

pression of PBX1 and EXD, even at high levels, always showed their spontaneous nuclear localization in mouse fibroblast and other tested mammalian cell lines. In this respect, it can be considered unlikely that the endogenous levels of PREP1 and/or MEIS in these cells mediate nuclear localization of highly expressed, exogenous PBX1 or EXD in a nonsaturable manner. Several mechanisms could be envisaged to explain the differential, cell context-dependent subcellular distribution of PBC proteins. One possibility is that the putative NES localized within the PBC-A domain is differentially modified in various cell contexts, such as to alter its capability to interact with the nuclear export receptor. Several examples of post-translational modifications involving NESs were recently reported (for review, see Mattaj and Englmeier 1998).

Nuclear export of EXD and PBX could be required in specific contexts, such as in distal Drosophila limb imaginal disc cells and in mouse distal limb mesenchymal cells to prevent EXD and PBX from inappropriately regulating target genes in space and time, in the absence of HTH and PREP1/MEIS proteins. It was shown that forcing EXD nuclear localization through overexpression in the distal regions of the Drosophila leg imaginal disc leads to a block in development of distal structures, due to a repression of genes downstream of Dpp and Wg signaling (Gonzalez-Crespo and Morata 1996; Abu-Shaar and Mann 1998).

In conclusion, our results indicate that cytoplasmic localization of $\mathrm{PBC}$ proteins, as observed in spatially re- stricted regions during Drosophila and mouse development, as well as in Schneider cells, is based on an active nuclear export mechanism operating on conserved sequences located within the PBC-A domain. On the basis of our data, we propose a model (Fig. 4), for the induction of nuclear localization of PBC proteins by PREP1/ MEIS1/HTH proteins, in cells that actively export PBC proteins from the nucleus. Heterodimerization with PREP1/MEIS1/HTH proteins, involving sequences overlapping the NES, would cause its inactivation, allowing NLS function to prevail, thereby leading to a shift in the nucleocytoplasmic shuttling equilibrium in favor of nuclear import. According to our model, PREP1/MEIS1/ $\mathrm{HTH}$ proteins, rather than acting as escorts for nuclear localization of PBC proteins, would trigger their nuclear translocation in specific cell contexts by antagonizing export from the cell nucleus.

\section{Materials and methods}

Expression constructs

Mammalian expression constructs for PREP1, PREPAHR1+2, PBX1, HA-PBX1, and PBX $\Delta 1-140$ were described previously (Di Rocco et al. 1997; Berthelsen et al. 1998b). The pSGFLAG-EXD and pSGFLAG-HTH expression constructs were generated by fusing the PCR-amplified ORFs of EXD or HTH to the Flag epitope and cloned into the pSG5 expression vector. pSGPbxNT was generated by PCR amplification of the region encoding amino acids 1-230 of Pbx1 and cloning into pSG5. The PREP/ PBXHD fusion contains residues 1-257 of PREP1, linked to the 


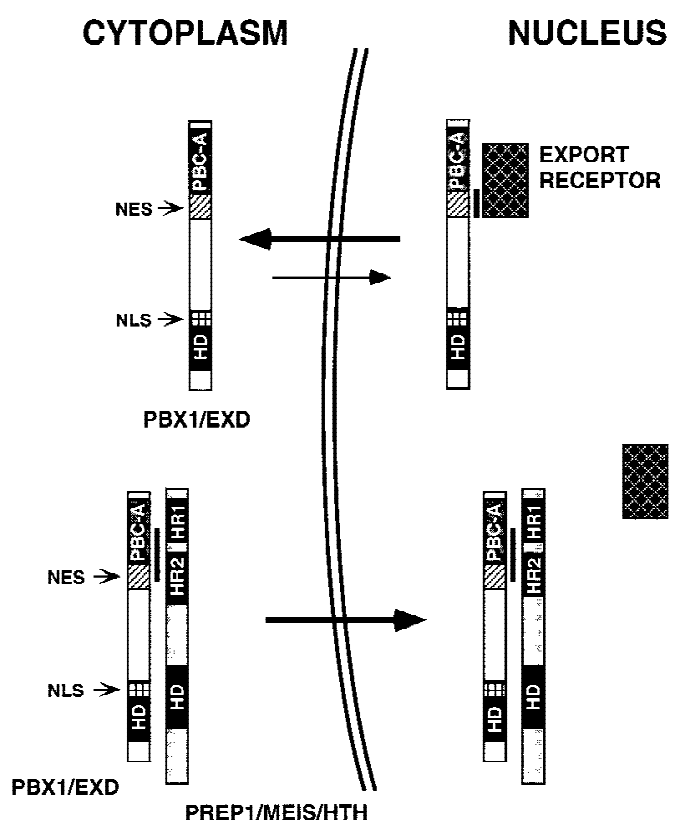

Figure 4. A model for the regulation of subcellular localization of PBC and PREP1/MEIS/HTH proteins in cells displaying cytoplasmic localization of PBC proteins. PBC and PREP1/MEIS/ $\mathrm{HTH}$ proteins are represented schematically. In specific cell contexts (e.g., Schneider cells), in the absence of PREP1/MEIS/ $\mathrm{HTH}$ proteins, $\mathrm{PBC}$ proteins are actively exported from the nucleus, a process requiring sequences (NES, hatched box) located within their conserved PBC-A domain, which are recognized by a nuclear export receptor (dark, squared rectangle). PBC proteins form stable complexes with PREP1/MEIS/HTH proteins, when coexpressed, through an interaction surface that coincides with the region required for nuclear export, thereby shielding it. The newly formed complex translocates into the nucleus owing to the NLS located within the homeodomain of PBC proteins (NLS, white-squared box). Black rectangles represent the homeodomains (HD). Light gray and dark gray boxes represent conserved amino-terminal regions within $\mathrm{PBC}$ and PREP1/MEIS/HTH proteins, respectively (PBC-A, HR1/HR2). A black vertical line indicates protein-protein contacts.

homeodomain and carboxy-terminal sequences of PBX1a (residues 232-431). The PBX1/PREPHD fusion contains residues 1-231 of PBX1a, linked to the homeodomain and carboxyl terminus of PREP1 (residues 258-436). Both chimeras are cloned into the pSG5 vector. The pSGNLSPrep1 expression construct was generated by cloning the Prep1 ORF downstream of an oligonucleotide encoding for the SV40 large T NLS into pSG5.

For expression in Drosophila Schneider cells, the cDNAs encoding PREP1, PBX1, Flag-EXD, and Flag-HTH were excised from the corresponding pSG5 expression constructs and cloned into the pAC5c actin promoter-driven expression vector. The $\mathrm{pACPbx} \Delta 1-140$ construct was generated excising the insert of pSGPbx $\Delta 1-140$ and cloning into pAC5c. The pACPbx $\Delta 1-72$ construct was generated by cloning a PCR product representing the Pbx mutant derivative into pAC5c. All PCR-derived constructs were verified by sequencing.

\section{Transfections and $L M B$ treatment}

NIH-3T3 fibroblast cells were grown in DMEM supplemented with $10 \%$ newborn calf serum and antibiotics. Cells were trans- fected with 5 or $10 \mu \mathrm{g}$ of the various mammalian expression constructs by $\mathrm{CaPO}_{4}$ precipitation in $10 \mathrm{~cm}$ dishes. Cells were reseeded after $24 \mathrm{hr}$ on Chambers Slides (Nunc) and fixed after $16 \mathrm{hr}$ for immunocytochemistry. Drosophila SL-2 Schneider cells were transfected with SuperFect (QIAGEN) according to the manufacturer's instructions. Transfected Drosophila SL-2 Schneider cells were treated $18 \mathrm{hr}$ after addition of DNA with 5, 50 , and $250 \mathrm{nM} \mathrm{LMB}$ for $0.5-6 \mathrm{hr}$ prior to fixation with methanol.

\section{Antibodies and immunocytochemistry}

Anti-PREP1 antibody (Berthelsen et al. 1998b) was used to detect wild-type PREP1, PREP $\triangle \mathrm{HR} 1+2$, and NLS-PREP1. Antibodies against PBX proteins were obtained from Santa Cruz Biotechnology (Santa Cruz, CA). The antibody $\alpha$ PBX1 C-20 recognizes an epitope in the carboxyl terminus of PBX1, and was used according to the manufacturer's instructions to detect the wildtype PBX1, HA-tagged PBX1, PREP/PBXHD, PBX $\Delta 1-72$, and PBX $\Delta 1-140$. The antibody $\alpha$ PBX1 P-20 recognizes an epitope in the amino terminus of $\mathrm{PBX} 1$, and was used to detect wild-type PBX1, HA-tagged PBX1, PBXNT, and PBX/PREPHD. Mouse monoclonal M2 Anti-Flag epitope antibodies (Sigma) was used according to the manufacturer's instructions to detect FlagEXD and Flag-HTH. Rat high affinity anti-HA tag (Boehringer) was used according to the manufacturer's instructions to detect HA-PBX1, and HA-PBXNT. Anti-rabbit, anti-mouse, or anti-rat FITC or TRITC-conjugated secondary antibodies (Sigma) were used according to the manufacturer's instructions. For immunocytochemistry, cells were fixed in 100\% methanol, rehydrated in PBS, blocked in PBS 1\% BSA, incubated for $1 \mathrm{hr}$ with the primary antibody, washed in PBS and $1 \%$ BSA, incubated for 1 hr with secondary antibody, washed with PBS and $1 \%$ BSA, and mounted for examination with an Olympus Provis fluorescence microscope.

\section{Acknowledgments}

We thank Henry Sun for providing us with the $h$ th cDNA, Barbara Wolff (Novartis) for providing LMB, Valerio Orlando for the kind gift of Schneider cells and for advice on their culturing, and Vincenzo Zimarino for support and helpful discussion. This work was supported by grants from the Italian Association for Cancer Research (AIRC) to V.Z. and F.B. and by a Telethon core grant. J.B. is supported by a fellowship from the Danish Research Academy.

The publication costs of this article were defrayed in part by payment of page charges. This article must therefore be hereby marked 'advertisement' in accordance with 18 USC section 1734 solely to indicate this fact.

\section{References}

Abu-Shaar, M. and R. Mann. 1998. Generation of multiple antagonistic domains along the proximodistal axis during Drosophila leg development. Development 125: 3821-3830.

Berthelsen, J., V. Zappavigna, E. Ferretti, F. Mavilio, and F. Blasi. 1998a. The novel homeoprotein Prep1 modulates Pbx-Hox protein cooperativity. EMBO J. 17: 1434-1445.

Berthelsen, J., V. Zappavigna, F. Mavilio, and F. Blasi. 1998b. Prep1, a novel functional partner of Pbx proteins. EMBO $J$. 17: 1423-1433.

Burglin, T. 1997. Analysis of TALE superclass homeobox genes (MEIS, PBC, KNOX, Iroquois, TGIF) reveals a novel domain conserved between plants and animals. Nucleic Acids Res. 
25: $4173-4180$.

Burglin, T.R. and G. Ruvkun. 1992. New motif in PBX genes. Nat. Genet. 1: 319-320.

Casares, F. and R.S. Mann. 1998. Control of antennal versus leg development in Drosophila. Nature 392: 723-726.

Chang, C.P., Y. Jacobs, T. Nakamura, N.A. Jenkins, N.G. Copeland, and M.L. Cleary. 1997. Meis proteins are major in vivo DNA binding partners for wild-type but not chimeric Pbx proteins. Mol. Cell. Biol. 17: 5679-5687.

Derossi, D., G. Chassaing, and A. Prochiantz. 1998. Trojan peptides: The penetratin system for intracellular delivery. Trends Cell Biol. 8: 84-87.

Di Rocco, G., F. Mavilio, and V. Zappavigna. 1997. Functional dissection of a transcriptionally active, target-specific HoxPbx complex. EMBO J. 16: 3644-3654.

Gonzalez-Crespo, S. and G. Morata. 1996. Genetic evidence for the subdivision of the arthropod limb into coxopodite and telopodite. Development 122: 3921-3928.

Gonzales-Crespo, S., M. Abu-Shaar, M. Torres, C. MartinesArias, R. Mann, and G. Morata. 1998. Antagonism between extradenticle function and Hedgehog signalling in the developing limb. Nature 394: 196-200.

Kalderon, D., B.L. Roberts, W.D. Richardson, and A.E. Smith. 1984. A short amino acid sequence able to specify nuclear location. Cell 39: 499-509.

Kamps, M.P., C. Murre, X.-H. Sun, and D. Baltimore. 1990. A new homeobox gene contributes the DNA binding domain of the $\mathrm{t}(1 ; 19)$ translocation protein in Pre-B-ALL. Cell 60: $547-555$.

Knoepfler, P.S., K.R. Calvo, H. Chen, S.E. Antonarakis, and M.P. Kamps. 1997. Meis1 and pKnoxl bind DNA cooperatively with Pbx1 utilizing an interaction surface disrupted in oncoprotein E2a-Pbx1. Proc. Natl. Acad. Sci. 94: 14553-14558.

Kurant, E., C.Y. Pai, R. Sharf, N. Halachmi, Y.H. Sun, and A. Salzberg. 1998. Dorsotonals/homothorax, the Drosophila homologue of meis1, interacts with extradenticle in patterning of the embryonic PNS. Development 125: 1037-1048.

Maconochie, M.K., S. Nonchev, M. Studer, S.K. Chan, H. Popperl, M.H. Sham, R.S. Mann, and R. Krumlauf. 1997. Crossregulation in the mouse HoxB complex: The expression of Hoxb2 in rhombomere 4 is regulated by Hoxb1. Genes \& Dev. 11: 1885-1895.

Mann, R.S. and S.-K. Chan. 1996. Extra specificity from extradenticle: The partnership between HOX and PBX/EXD homeodomain proteins. Trends Genet. 12: 258-262.

Mattaj, I.M. and L. Englmeier. 1998. Nucleocytoplasmic transport: The soluble phase. Annu. Rev. Biochem. 67: 265-306.

Moskow, J., F. Bullrich, K. Huebner, I. Daar, and A. Buchberg. 1995. Meis1, a PBX1-related homeobox gene involved in myeloid leukemia in BXH-2 mice. Mol. Cell. Biol. 15: 5434 5443.

Ohno, M., M. Fornerod, and I.W. Mattaj. 1998. Nucleocytoplasmic transport: The last 200 nanometers. Cell 92: 327-336.

Pai, C.Y., T.S. Kuo, T.J. Jaw, E. Kurant, C.T. Chen, D.A. Bessarab, A. Salzberg, and Y.H. Sun. 1998. The Homothorax homeoprotein activates the nuclear localization of another homeoprotein, extradenticle, and suppresses eye development in Drosophila. Genes \& Dev. 12: 435-446.

Rieckhof, G.E., F. Casares, H.D. Ryoo, M. Abu-Shaar, and R.S. Mann. 1997. Nuclear translocation of extradenticle requires homothorax, which encodes an extradenticle-related homeodomain protein. Cell 91: 171-183. 


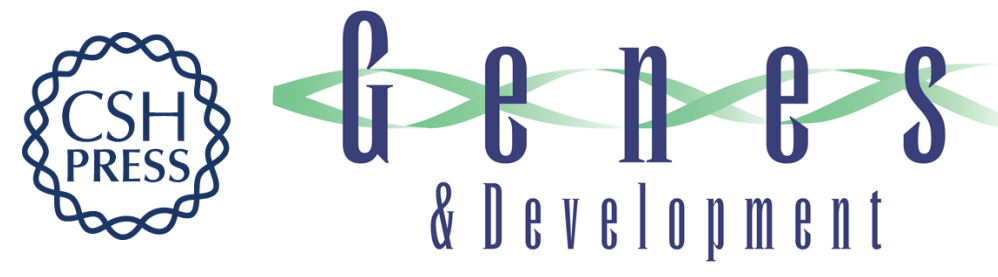

\section{The subcellular localization of PBX1 and EXD proteins depends on nuclear import and export signals and is modulated by association with PREP1 and HTH}

Jens Berthelsen, Charlotte Kilstrup-Nielsen, Francesco Blasi, et al.

Genes Dev. 1999, 13:

References This article cites 22 articles, 11 of which can be accessed free at: http://genesdev.cshlp.org/content/13/8/946.full.html\#ref-list-1

License

Email Alerting Receive free email alerts when new articles cite this article - sign up in the box at the top Service right corner of the article or click here.

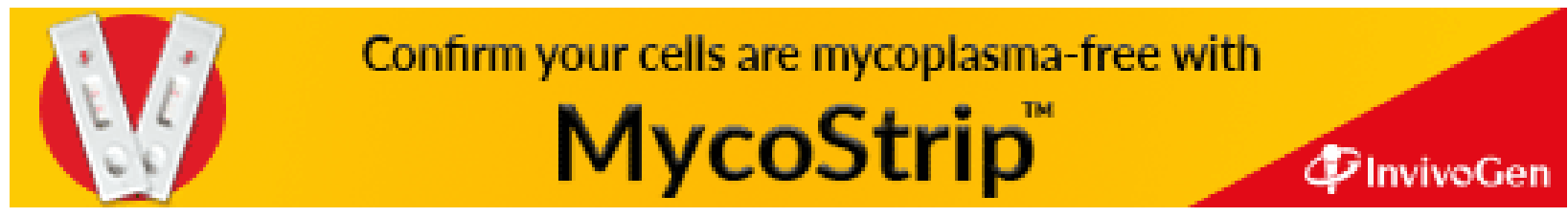

